\title{
A síndrome de Burnout em enfermeiros frente a pandemia: uma revisão integrativa
}

\section{da literatura}

\author{
Burnout's syndrome in nurses in front of the pandemic: an integrative literature review \\ Síndrome de Burnout en enfermeras frente a la pandemia: revisión integradora de la literatura
}

Recebido: 08/10/2021 | Revisado: 15/10/2021 | Aceito: 20/10/2021 | Publicado: 22/10/2021

Mayara Fernanda Angotti Reese

ORCID: https://orcid.org/0000-0001-8446-3755 Centro Universitário Dinâmica das Cataratas, Brasil

E-mail: mayara_reese@outlook.com

Andressa Edyla Kuntz Linden

ORCID: https://orcid.org/0000-0003-4077-4951 Centro Universitário Dinâmica das Cataratas, Brasil E-mail:dre_linden@hotmail.com

Wesley Martins

ORCID: https://orcid.org/0000-0003-1083-9515 Centro Universitário Dinâmica das Cataratas, Brasil E-mail:wesley.martins@udc.edu.br

\begin{abstract}
Resumo
Objetivo: identificar na literatura cientifica os fatores predisponentes para o desenvolvimento da síndrome de Burnout em profissionais da enfermagem durante a pandemia do COVID-19. Metodologia: trata-se de uma revisão integrativa da literatura realizada por meio dos estudos disponíveis na Biblioteca Virtual em Saúde, Literatura Latino Americana e do Caribe em ciências da saúde e no portal Scientific Eletronic Library Online. Os critérios estabelecidos como inclusão nessa pesquisa foram: estudos completos e originais disponibilizados gratuitamente, período de publicação entre os últimos três anos (2019 a 2021) no idioma português. Foram utilizados os seguintes descritores: "Síndrome de Burnout"; "Enfermagem"; "COVID 19" para a eleição dos artigos científicos. Os dados foram analisados de forma descritiva. Resultados: foram identificados dez artigos, separados em três categorias: estudos com foco na sobrecarga de trabalho, estudos com foco nos preditores do adoecimento mental e estudos com foco no estado clinico dos pacientes. Conclusão: os sinais mais destacados entre os profissionais foram a depressão e angústia, por estarem continuamente lidando com as demandas de exigências dos pacientes que são complexos e graves. Além disso, os estudos levantaram que essa síndrome pode ser desencadeada por fatores associados ao ambiente de trabalho, como a falta de recursos humanos, de estrutura física e de valorização por parte dos gestores e empregadores.
\end{abstract}

Palavras-chave: Síndrome de Burnout; Enfermagem; COVID-19.

\begin{abstract}
Objective: to identify in the scientific literature the predisposing factors for the development of Burnout syndrome in nursing professionals during the COVID-19 pandemic. Methodology: this is an integrative literature review carried out through the studies available in the Virtual Health Library, Latin American and Caribbean Literature in Health Sciences and in the Scientific Electronic Library Online portal. The criteria established for inclusion in this research were: complete and original studies made available for free, publication period between the last three years (2019 to 2021) in Portuguese. The following descriptors were used: "Burnout Syndrome"; "Nursing"; "COVID 19" for the election of scientific articles. Data were analyzed descriptively. Results: ten articles were identified, divided into three categories: studies focusing on workload, studies focusing on predictors of mental illness and studies focusing on the clinical status of patients. Conclusion: the most prominent signs among professionals were depression and anxiety, as they are continually dealing with the demands of patients' demands that are complex and severe. In addition, studies have shown that this syndrome can be triggered by factors associated with the work environment, such as the lack of human resources, physical structure and appreciation on the part of managers and employers.
\end{abstract}

Keywords: Burnout Syndrome; Nursing; COVID-19.

\section{Resumen}

Objetivo: identificar en la literatura científica los factores predisponentes para el desarrollo del síndrome de Burnout en profesionales de enfermería durante la pandemia de COVID-19. Metodología: se trata de una revisión integradora de la literatura realizada a través de los estudios disponibles en la Biblioteca Virtual en Salud, la Literatura Latinoamericana y del Caribe en Ciencias de la Salud y el portal de la Biblioteca Electrónica Científica en Línea. Los criterios establecidos para la inclusión en esta investigación fueron: estudios completos y originales disponibles de forma gratuita, período de 
publicación entre los últimos tres años (2019 a 2021) en portugués. Se utilizaron los siguientes descriptores: "Síndrome de Burnout"; "Enfermería"; "COVID 19" para la elección de artículos científicos. Los datos se analizaron de forma descriptiva. Resultados: se identificaron diez artículos, divididos en tres categorías: estudios centrados en la carga de trabajo, estudios centrados en predictores de enfermedad mental y estudios centrados en el estado clínico de los pacientes. Conclusión: los signos más destacados entre los profesionales fueron la depresión y la ansiedad, ya que continuamente están lidiando con las demandas complejas y severas de los pacientes. Además, los estudios han demostrado que este síndrome puede desencadenarse por factores asociados al entorno laboral, como la falta de recursos humanos, estructura física y valoración por parte de directivos y empresarios.

Palabras clave: Síndrome de Burnout; Enfermería; COVID-19.

\section{Introdução}

Com a evidência da pandemia pelo novo coronavírus (SARS-COV-2), agente da doença denominada COVID-19, muitas publicações foram sendo produzidas com o intuito de esclarecer e orientar a população do mundo, especialmente quem trabalha na área da saúde, quais seriam os meios de contágio da doença e as formas de prevenção (Anvisa, 2020).

Ainda de acordo com a citação supracitada, a demanda de trabalho de urgência que foram estabelecidas em todos os hospitais do mundo gerou um grande número de informações entre artigos científicos, normas técnicas, manuais, reportagens, boletins informativos que tiveram que ser filtradas, interpretadas e transformadas em ações pontuais pra que pudessem atender o grande impacto e os desafios dos serviços de saúde de todo o planeta.

Sendo assim, Xavier et al., (2020) destacam que os dados colhidos do paciente, associados aos cuidados clínicos e de todos os profissionais de saúde, são ferramentas muito importantes e primordiais para que o diagnóstico da doença seja estabelecido. Também, por meio desses detalhes no primeiro atendimento ao paciente, é possível acompanhar a evolução da doença, entender como pode ser o prognóstico e estabelecer critérios para o tratamento de outros indivíduos.

Essas mudanças repentinas causadas no mundo, afetaram e desestruturaram todas as instituições de saúde, que passaram a solicitar muito mais atenção e cuidados em relação aos pacientes que estão sob estado crítico. Nesta realidade, há uma exigência maior em relação a capacitação desses profissionais, especialmente o enfermeiro que se encontra na linha de frente das instituições, especialmente nas áreas de emergência e terapia intensiva (Paiva et al., 2016).

Períodos longos de exaustão no ambiente laboral causam o estresse considerado crônico. Nesta perspectiva os profissionais enfermeiros deixam de lado a sua vida individual. Ao longo da jornada difícil de enfrentamentos diante da pandemia, ainda devem conviver com os riscos de adquirir a doença ou de transmiti-la para os seus familiares. São situações que podem trazer sentimentos de insatisfação e tristeza continuam desencadeando a falta de ânimo para a execução das atividades diárias (Luz et al., 2017).

Como profissionais enfermeiros precisam e desdobrar para conviver com a realidade presente, poderá estar bem próximo de sinais e sintomas que denotam a Síndrome de Burnout. A doença pode ser vista como um problema de saúde pública. O número de pessoas que relatam sintomas relacionados a doença vem aumentando gradativamente ao logo dos tempos. Especialmente no contexto da pandemia, a falta de oportunidade para olhar para si mesmo, faz com o enfermeiro não dê importância para reações do próprio corpo que mostram que algo não vai bem, como insônia, cansaço excessivo, dores de cabeça, entre outros. Todos esses cenários de conflito levam a prejuízos relacionados a qualidade de vida dos profissionais, seja fora ou dentro do ambiente de trabalho (Baldonedo et al., 2019).

Diante desta problemática que envolve profissionais enfermeiros e a dinâmica de trabalho e de vida comprometidos, devido ao desgaste pelos atendimentos aos pacientes críticos consequentes da Covid 19, surge o seguinte questionamento que norteou este estudo: "Quais os fatores predisponentes para o desenvolvimento da síndrome de Burnout em profissionais da enfermagem durante a pandemia do COVID-19?”.

Dessa forma, essa pesquisa objetivou identificar na literatura cientifica os fatores predisponentes para o desenvolvimento da síndrome de Burnout em profissionais da enfermagem durante a pandemia do COVID-19. 


\section{Metodologia}

Trata-se de um estudo de revisão integrativa da literatura, pela qual permite a construção de uma análise ampla da literatura, contribuindo para discussões sobre métodos e resultados de pesquisas, assim como reflexões sobre a realização de futuros estudos. Tal pesquisa identificou os fatores predisponentes para o desenvolvimento da síndrome de Burnout em profissionais da enfermagem durante a pandemia do COVID-19.

A pesquisa foi realizada por meio dos estudos disponíveis na Biblioteca Virtual em Saúde (BVS-BIREME), nas bases de dados Literatura Latino Americana e do Caribe em ciências da saúde (LILACS) e no portal Scientific Eletronic Library On line (SCIELO). A coleta de dados ocorreu no período de abril a julho de 2021.

Os critérios estabelecidos como inclusão nessa pesquisa foram: estudos completos e originais disponibilizados gratuitamente nesses bancos de dados previamente estabelecidos. Também foi estipulado o período de publicação entre os últimos três anos (2019 a 2021), assim como estar publicado no idioma português.

Para a construção desta revisão integrativa da literatura, optou-se por adotar as etapas estabelecidas pelo método de Gil (2010). A seguir, serão descritos os procedimentos que utilizaremos:

$1^{\text {a: }}$ Identificação do tema e seleção da hipótese ou questão da pesquisa para elaboração da pesquisa integrativa.

$2^{\mathrm{a}}$ : Estabelecimento de critérios para a inclusão e exclusão de estudos/amostragem ou busca na literatura.

$3^{\text {a }}$ : Definição das informações a serem extraídas dos estudos selecionados/categorização dos estudos.

$4^{\mathrm{a}}$ : Avaliação dos estudos.

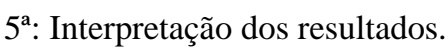

6: Apresentação da revisão/síntese do conhecimento.

Nos bancos de dados previamente estabelecidos, foram utilizados os seguintes descritores: "Síndrome de Burnout"; "Enfermagem"; "COVID 19" para a eleição dos artigos científicos.

Ressalta-se que em primeiro momento foram analisados os títulos e resumos de cada artigo, a fim de realizar uma primeira filtragem dos estudos relacionados ao tema proposto.

Após essa primeira seleção, os artigos selecionados passaram para análise completa, na qual as pesquisadoras analisaram a pertinência do estudo e a relação com a pergunta de pesquisa, totalizando somente os artigos que consigam responder à questão norteadora. Os dados levantados nessa pesquisa foram analisados de forma descritiva.

Por se tratar de um estudo de revisão integrativa da literatura, esse estudo não passou por análise do Comitê de Ética em Pesquisa Envolvendo Seres Humanos (CEP), visto que nenhum dado individualizado foi levantado, todavia as pesquisadoras se comprometam em respeitar todas as questões éticas e legais regidos nas resoluções CNS 466/2012 e CNS 510/2015.

\section{Resultados e Discussão}

Medeiros et al. (2018) destacam que os enfermeiros vivem momento de desgastes tanto físico como emocionais, porém na medida em que se envolvem com o trabalho, buscam alternativas em como dividir as preocupações com outros colegas no sentido de dividir a sobrecarga de trabalho presente nesta pandemia.

A busca por ajuda e valorização no ambiente de trabalho, sobrecarregado de tarefas tendem a fazer com que muitos profissionais se sintam desanimados e cansados mentalmente. Neste panorama de trabalhos excessivos, a Síndrome de Burnout pode acometer estes indivíduos e fazer com que desenvolvam um estresse que passa despercebido devido a correria e as exigências da situação (Medeiros et al., 2018).

No Quadro 1 estão reunidos os artigos conforme a seleção. Estão organizados de acordo com as seguintes variáveis: 
Número do artigo, Título do estudo, Autores, Revista, e Ano de publicação e Objetivo.

Quadro 1 - Distribuição dos artigos conforme as variáveis: Número do artigo, Título do estudo, Autores, Revista e ano de publicação, Objetivo e Tipo de estudo - Paraná, 2021.

\begin{tabular}{|c|c|c|c|c|}
\hline Art. & Título & Autores & Revista / ano & Objetivo \\
\hline 01 & $\begin{array}{l}\text { Estresse ocupacional de } \\
\text { enfermeiros: uma visão crítica em } \\
\text { tempos de pandemia }\end{array}$ & Miranda, A. & $\begin{array}{l}\text { Brazilian Journal of } \\
\text { Development } \\
2020\end{array}$ & $\begin{array}{l}\text { Abordar o impacto das condições de trabalho } \\
\text { sobre o estresse ocupacional dos } \\
\text { enfermeiros, por meio de uma revisão de } \\
\text { literatura }\end{array}$ \\
\hline 02 & $\begin{array}{l}\text { Proteção da saúde dos } \\
\text { trabalhadores da saúde em tempos } \\
\text { de pandemia e respostas à } \\
\text { pandemia }\end{array}$ & Almeida, I. M & $\begin{array}{c}\text { Revista brasileira de } \\
\text { saúde ocupacional } \\
2020\end{array}$ & $\begin{array}{l}\text { Discutir desafios para a elaboração e } \\
\text { implementação de planos de resposta } \\
\text { prontidão estratégica contra a COVID-19 }\end{array}$ \\
\hline $\mathbf{0 3}$ & $\begin{array}{l}\text { Sofrimento emocional dos } \\
\text { Enfermeiros no contexto } \\
\text { hospitalar frente à pandemia de } \\
\text { COVID-19 }\end{array}$ & Pereira, M. D., et al. & $\begin{array}{l}\text { Research, Society } \\
\text { and Development. } \\
2020\end{array}$ & $\begin{array}{l}\text { Realizar uma revisão teórica sobre o } \\
\text { sofrimento emocional dos enfermeiros no } \\
\text { contexto hospitalar frente à pandemia de } \\
\text { COVID-19 }\end{array}$ \\
\hline 04 & $\begin{array}{l}\text { Fatores de risco para a Síndrome } \\
\text { de Burnout em profissionais da } \\
\text { saúde durante a pandemia de } \\
\text { COVID-19 }\end{array}$ & Borges, F. E., et al. & $\begin{array}{c}\text { Revista } \\
\text { Enfermagem } \\
\text { Atual In Derme } \\
2021 \\
\end{array}$ & $\begin{array}{l}\text { Analisar os fatores de risco para } \\
\text { desenvolvimento da Síndrome de Burnout } \\
\text { em profissionais da saúde durante a } \\
\text { pandemia da COVID -19 }\end{array}$ \\
\hline 05 & $\begin{array}{l}\text { COVID-19 e saúde mental: a } \\
\text { emergência do cuidado }\end{array}$ & Faro, A., et al. & $\begin{array}{c}\text { Estudos Psicologia } \\
2020\end{array}$ & $\begin{array}{l}\text { Reunir informações e achados de pesquisa a } \\
\text { respeito do impacto de tais crises na saúde } \\
\text { mental }\end{array}$ \\
\hline 06 & $\begin{array}{l}\text { A saúde mental da enfermagem no } \\
\text { enfrentamento da COVID-19 em } \\
\text { um hospital universitário regional }\end{array}$ & $\begin{array}{l}\text { Dal'Bosco, E. B., et } \\
\text { al. }\end{array}$ & $\begin{array}{l}\text { Revista Brasileira de } \\
\text { Enfermagem } \\
2020\end{array}$ & $\begin{array}{l}\text { Identificar a prevalência e fatores associados } \\
\text { à ansiedade e depressão em profissionais de } \\
\text { enfermagem que atuam no enfrentamento da } \\
\text { COVID-19 em hospital universitário. }\end{array}$ \\
\hline $\mathbf{0 7}$ & $\begin{array}{l}\text { A saúde mental dos profissionais } \\
\text { de saúde frente à pandemia do } \\
\text { COVID-19: uma revisão } \\
\text { integrativa }\end{array}$ & Prado, A. D., & $\begin{array}{l}\text { Revista Eletrônica } \\
\text { Acervo Saúde } \\
2020\end{array}$ & $\begin{array}{l}\text { Conhecer a situação da saúde mental dos } \\
\text { profissionais da área da saúde da linha de } \\
\text { frente na pandemia do COVID -19, e quais } \\
\text { consequências para os serviços de saúde. }\end{array}$ \\
\hline 08 & $\begin{array}{l}\text { Preditores da síndrome de Burnout } \\
\text { em técnicos de enfermagem de } \\
\text { unidade de terapia intensiva } \\
\text { durante a pandemia da COVID-19 }\end{array}$ & Freitas, R. F., & $\begin{array}{l}\text { Journal Brasileiro de } \\
\text { Psiquiatria } \\
2021\end{array}$ & $\begin{array}{l}\text { Avaliar a prevalência e a existência de } \\
\text { fatores preditores da síndrome de Burnout } \\
\text { em técnicos de enfermagem que atuam em } \\
\text { unidade de terapia intensiva (UTI) durante a } \\
\text { pandemia da COVID-19 }\end{array}$ \\
\hline 09 & $\begin{array}{l}\mathrm{O} \text { estresse e a saúde mental de } \\
\text { profissionais da linha de frente da } \\
\text { COVID-19 em hospital geral }\end{array}$ & Horta, R. L., et al. & $\begin{array}{c}\text { Journal Brasileiro de } \\
\text { Psiquiatria } \\
2021 \\
\end{array}$ & $\begin{array}{l}\text { Investigar os efeitos da atuação na linha de } \\
\text { frente da COVID-19 na saúde mental de } \\
\text { profissionais de hospital público }\end{array}$ \\
\hline 10 & $\begin{array}{l}\text { Saúde mental de enfermeiros } \\
\text { durante a pandemia }\end{array}$ & $\begin{array}{l}\text { Santos, K. M. R., et } \\
\text { al. }\end{array}$ & $\begin{array}{c}\text { Escola Anna Nery } \\
2021\end{array}$ & $\begin{array}{l}\text { Analisar a prevalência de sintomas } \\
\text { depressão, ansiedade e fatores associados em } \\
\text { profissionais da equipe de enfermagem } \\
\text { durante a pandemia da Covid-19 }\end{array}$ \\
\hline
\end{tabular}

Fonte: Coleta de dados.

Diante dos dez estudos levantados, elencamos três categorias distintas para discussão, sendo elas: estudos com foco na sobrecarga de trabalho; estudos com foco nos preditores do adoecimento mental; e estudos com foco no estado clínicos dos pacientes (Tabela 1).

Tabela 1 - Principias fatores que propiciam a síndrome de burnout nos enfermeiros em tempos de pandemia - Brasil, 2021. 
Tabela 1 - Principias fatores que propiciam a síndrome de burnout nos enfermeiros em tempos de pandemia - Brasil, 2021.

\begin{tabular}{llc}
\hline \multicolumn{1}{c}{ CATEGORIAS } & N & ARTIGOS \\
\hline Estudos com foco na sobrecarga de trabalho & 05 & A1; A2; A3; A4; A5 \\
Estudos com foco nos preditores do adoecimento mental & 03 & A6; A7; A8 \\
Estudos com foco no estado clínico dos pacientes & 02 & A9; A10 \\
\hline
\end{tabular}

Fonte: Coleta de dados.

Quanto as categorias criadas de acordo com os resultados encontrados, percebe-se que 50\% dos estudos focaram na sobrecarga de trabalho, enquanto $30 \%$ abordaram os preditores do adoecimento mental e $20 \%$ no estado clinico dos pacientes. A seguir serão discutidos separadamente cada categoria.

\section{Estudos com foco na sobrecarga de trabalho}

Ao abordar a categoria direcionada a sobrecarga de trabalho, pôde-se levantar os artigos A1; A2; A3; A4 e A5.

O estudo A1 objetivou abordar o impacto das condições de trabalho sobre a geração de estresse nos enfermeiros, por meio de uma revisão de literatura. Sendo assim, o propósito dos autores em compreender quais os fatores que desencadeiam o estresse e suas consequências na saúde do trabalhador especialmente na pandemia, pode envolver especialmente aqueles que lidam rotineiramente com o cuidado intensivo. Este estudo, trouxe evidências no decorrer do seu desenvolvimento sobre a preocupação com questões laborais e a saúde do trabalhador que foi intensificada com a pandemia, enfatizando quais seriam as necessidades de melhorias no ambiente de trabalho e as condições de trabalho.

O impacto à saúde causado pela pandemia, fez refletir sobre as mudanças no mundo do trabalho. Essas mudanças vieram associadas a grande carga tecnológica que os profissionais devem dar conta no seu dia de trabalho, além de processos de gestão, cobranças e relações socias no ambiente laboral. São assuntos discutidos e presentes na Lei Federal 8.080, que criou o Sistema Único de Saúde (SUS) que aborda sobre esta temática, focando nas ações de vigilância epidemiológica e sanitária, promoção, recuperação e reabilitação dos profissionais segundo as suas condições de trabalho. Os autores destacam que apesar dessa os profissionais de enfermagem permanecem expostos aos riscos de adoecimento tanto físico quanto mental.

Os autores Vidotti et al. (2020) destacam que, nestes momentos de desafios, os profissionais da equipe de enfermagem devem se comprometer a dar apoio uns aos outros, pois todos vivenciam os problemas em comum e as formas de tratamento mútuo deve seguir os princípios da igualdade e de respeito entre todos da equipe, independentemente da posição que ocupam. Quando o tratamento é igualitário entre todos, há menos chances de adoecimentos entre os profissionais.

O estudo A2 objetivou minimizar o impacto da epidemia sobre sistemas de saúde, por meio do levantamento dos fatores de risco que o trabalhador está sujeito, dentre eles a sobrecarga de trabalho, que pode levar a maior incidência de erros e acidentes, além da contaminação pelo uso inadequado de Equipamentos de Proteção Individual - EPIs. A lista de fatores de risco que influenciam para a sobrecarga psicológica do profissional inclui a realização das atividades e a correria influenciada pelas demandas diárias, momentos de cansaço e falta de apoio quando ocorre algo novo no cotidiano laboral e não há alguém próximo para sanar dúvidas. Este fato traz desequilíbrio no desempenho do profissional e o torna descontente com a sua performance.

Em relação ao adoecimento e ao tempo de trabalho, Galea (2014) menciona que quanto mais tempo na profissão, mais comprometidos com seu trabalho o profissional tende a ficar. Com isso, diminui-se as chances de resiliência no enfrentamento de situações imprevisíveis e estressoras, o que colabora na pouca manifestação de despersonalização devido a maturidade profissional adquirida ao longo dos anos. Podem assim tornar-se mais insensíveis e distantes, sendo essa uma forma de se protegerem da fadiga por compaixão e evitar sofrimento, ou seja, protegem sua saúde mental.

Quanto ao estudo A3, cujo o método foi a revisão de literatura, objetivou realizar uma revisão teórica sobre o sofrimento 
emocional dos enfermeiros no contexto hospitalar frente à pandemia de COVID-19. Os autores ressaltam que o ambiente hospitalar exige que o profissional de enfermagem realize suas atividades em ambientes carregados de experiências que o sobrecarregam emocionalmente e favorecem as manifestações de altos níveis de estresse. Situações como lidar com a morte e a dor continuamente, além das demandas de trabalho intensas, se tornam focos de surgimento da síndrome de Burnout nos trabalhadores.

Os autores evidenciam ainda as estratégias sugeridas aos enfermeiros para o enfrentamento da COVID 19. Dentro desta perspectiva, indicam que a redução de carga de trabalho e a possibilidade de aumentar os períodos de descanso, além de incentivar a comunicação efetiva, podem colaborar para que o profissional tenha suporte e apoio dentro da situação de pandemia. Citam que além disso, os profissionais podem ser encorajados a realizar intervenções em suas vidas como a meditação entre outros recursos que aliviarão o estresse emocional e consequentemente as chances de desenvolver a Síndrome de Burnout. o estudo conclui como uma alerta aos profissionais de enfermagem para que fique mais atentos aos sinais e sintomas que podem estar sendo negligenciados a longo prazo e que tendem a evoluir de sintomas leves a sintomas mais graves como os transtornos mentais.

Sobre isso, Schmidt et al. (2020) esclarecem que o COVID 19 trouxe um fator muito complicado para a vida dos profissionais que é o distanciamento obrigatório dos mesmos em relação a família, sendo este uma situação determinante do estresse, pois a falta de afeto pode prejudicar mais ainda o emocional desses trabalhadores, colocando-os mais vulneráveis a Síndrome de Burnout, podem ainda se sentirem inadequados, decepcionados e desvalorizados dentro da própria atuação como profissionais.

No estudo A4 em uma revisão de literatura, os autores buscaram analisar os fatores de risco para o desenvolvimento da Síndrome de Burnout em profissionais da saúde durante a pandemia da COVID -19. Deixam claro neste estudo o conceito de Síndrome de Burnout que é caracterizada por exaustão emocional, além de despersonalização e diminuição da realização pessoal. Sintomas que surgem frente as fontes que se tornam crônicas de estresse diário, muito comum entre os profissionais de enfermagem pelas demandas e sobrecargas de trabalho, especialmente nos momentos em que o mundo enfrenta os desafios dos cuidados aos pacientes com COVID 19. O medo de contrair a doença e de infecção dos familiares também são grandes causadores de estresse psicológico, notados pelos autores deste estudo.

Destacam ainda a importância de refletir sobre o sofrimento dos trabalhadores de enfermagem quando precisam realizar o distanciamento social e da própria família, fator de estresse relacionado também aos sentimentos de apoio inadequados pela desvalorização do profissional no ambiente laboral. Concluem sobre o repensar dos empregadores quanto a dimensão da exaustão emocional e a sua representatividade nos sintomas relacionados a Síndrome de Burnout pelo esgotamento físico e mental associado ao cotidiano de trabalho.

O objetivo da revisão de literatura A5 foi reunir informações e achados de pesquisa a respeito do impacto de tais crises na saúde mental. Sendo assim, evidenciam que, além do medo de contrair a COVID-19, as pessoas e especialmente os profissionais de saúde - enfermagem, se mostram inseguros em todos os aspectos da vida. Os autores enfatizam que, a saúde mental prejudicada como a realidade se mostra frente a situação da pandemia, pode ter mais sequelas negativas do que as mortes em todo o mundo. Os profissionais de saúde permanecem exaustos pelas longas horas de trabalho, o que impacta consideravelmente a saúde como um todo.

Rodrigues e Silva (2020) discutem que além da possibilidade e medo de infectar outras pessoas, os profissionais estão expostos às mortes de muitas pessoas continuamente. Assim essa vivência traz a frustração pela perda da vida dos pacientes que cuidam e seus pacientes bem como, ansiedade, depressão, insônia, estresse associados à dificuldade de adormecer e ao despertar matinal, falta de energia, comprometimento das relações sociais e medo.

Os autores do A5 ressaltam que, os esforços imediatos devem ser empregados para que, possam diminuir as chances de 
resultados negativos na saúde mental das pessoas, sobretudo nos profissionais que necessitam estar em condições de atuar frente aos desafios trazidos pelo cuidado ao COVID 19.

\section{Estudos com foco nos preditores do adoecimento mental}

O artigo A6 mostra um estudo observacional transversal, com aplicação de um questionário sociodemográfico e Escala de Medida de Ansiedade e Depressão em 88 profissionais de enfermagem, cujo objetivo foi identificar a prevalência e fatores associados à ansiedade e depressão em profissionais de enfermagem que atuam no enfrentamento da COVID-19 em hospital universitário.

De acordo com os autores, a realidade é que a enfermagem tem um contingente maior no número de profissionais de saúde. Todo trabalho dessa equipe é centralizado no indivíduo e suas necessidades humanas, por isso, há uma ligação mais direta entre pacientes e profissionais, o que pode expor de forma mais precisa os mesmos aos potenciais impactos negativos psicossociais e psicossomáticos gerados por esta relação de proximidade. Quando há a falta de recursos humanos nas equipes de trabalho, a sobrecarga afeta os trabalhadores presentes, o que acarreta a diminuição da produtividade e o aumento do índice de acidentes de trabalho, além de uma assistência de enfermagem ineficaz, estes são considerados preditores do adoecimento mental dos trabalhadores.

Reforçando o que destacam os autores A6, afirmam que o Conselho Federal de Enfermagem (COFEN) demonstra preocupação com os profissionais de enfermagem, pois além dos riscos psicológicos, os indivíduos podem não estar sendo devidamente amparados quanto ao uso eficaz dos EPIs conforme os protocolos determinados pelo Ministério da Saúde (MS). A capacitação desses profissionais que estão na linha de frente é uma garantia de que estejam preparados para enfrentar os desafios da doença e o que representa uma parcela dos desafios que se trata das barreiras físicas, pois a COVID 19 mostrou trazer cargas emocionais grandes e deixar vulnerável os trabalhadores de enfermagem. É preciso proteger e orientar para evitar que a doença dissemine ainda mais, o que já está sendo mostrado em evidências estatísticas quando se refere a saúde do trabalhador de enfermagem (Cofen, 2020).

Os autores do estudo A6 ressaltam outro ponto importante, pois grande de parte dos pacientes acometidos pela COVID19 apresentam-se gravemente doentes, o cuidado intensivo é a única saída. Nesta realidade a ansiedade é maior entre os trabalhadores de enfermagem a há outras demandas de outros fatores potencialmente estressores, como exposição ao risco de infecção pelo vírus pelas atividades e falta de profissionais para assumi-las em igual proporção, gerando fadiga física e mental, sintomas da síndrome de Burnout. Concluem que as pressões no trabalho, conflitos, responsabilidade técnica que as faltas de profissionais contribuem para o desequilíbrio emocional dos profissionais de enfermagem por serem preditores do adoecimento mental ao longo do tempo de exposição.

Oliveira et al. (2015) estudaram a distribuição de doenças psicológicas em profissionais de enfermagem e pode constatar que, a depressão, na maioria estava presente nos técnicos de enfermagem e nos enfermeiros em cargo assistencial, especialmente naqueles profissionais locados em unidades críticas e que possuíam contato mais próximo com os pacientes. Estes dados complementam os estudos A6 e A7, onde os autores discutem os fatores estressores e dentre eles a sobrecarga de trabalho que traz o desequilíbrio emocional dos profissionais muito próximos ao paciente.

O objetivo do estudo A7 foi conhecer a situação da saúde mental dos profissionais da área da saúde da linha de frente na pandemia do COVID -19, e quais consequências para os serviços de saúde. Se trata de uma revisão bibliográfica onde os autores citam que além da exaustão física e mental, a dor da perda de pacientes e colegas, associados a dificuldade de decidir traz como resultados o medo e a insegurança no processo de cuidado ao paciente com COVID 19 e prejudicam efetivamente a saúde mental dos profissionais, especialmente os que estão na linha de frente.

O estudo A8 objetivou avaliar a prevalência e a existência de fatores preditores da síndrome de Burnout em técnicos de 
enfermagem que atuam em unidade de terapia intensiva (UTI) durante a pandemia da COVID-19. Trata-se de um estudo descritivo, de caráter transversal e abordagem quantitativa com 94 técnicos de enfermagem de terapia intensiva. Os instrumentos utilizados foram: um formulário de coleta de dados sociodemográficos, ocupacionais e comportamentais e o Maslach Burnout Inventory (MBI) em sua versão Human Services Survey (HSS), as repostas quanto aos preditores da Síndrome de Burnout foram constatados pela prevalência da síndrome em $25,5 \%$ dos profissionais analisados. Estes indivíduos tinham a idade maior que 36 anos e realizavam horas extras de trabalho continuamente e adoeceram mentalmente por conviver com horas de trabalho exaustivas e rígidas sem opções de flexibilidade.

Mercês et al. (2017) acrescentam o estudo A8 acima, e descrevem que, a prevalência da SB aumenta com o avançar da idade, contradizendo os achados de outros estudos realizados anteriormente. Afirmam ainda que, os mais jovens não estão isentos da doença pois são inexperientes quanto a profissão e necessitam ser vistos com critérios, pois podem não reconhecer os sintomas e nem perceber que estão doentes. As pessoas tendem a desenvolver transtornos mentais quando não adaptadas às condições laborais

Neste estudo (A8) os componentes avaliados para desencadear o Burnout foram a exaustão emocional e despersonalização, a sobrecarga e os conflitos existentes no trabalho e a falta de valorização foram determinantes para que os achados pudessem ser reais e preocupantes. Diante de uma situação de pandemia onde os profissionais se encontram exauridos e extremamente envolvidos no trabalho se torna mais evidentes que a doença prevaleça e atinja o estado mental de forma negativa.

\section{Estudos com foco no estado clínico dos pacientes}

No estudo A9, os autores investigaram os efeitos da atuação na linha de frente da COVID-19 na saúde mental de profissionais de hospital público. Os resultados revelaram que os profissionais destacaram como dificuldades especialmente nesta fase de pandemia, os longos e estressantes plantões, sem intervalos para descanso, o aumento das pressões inerentes ao ambiente de trabalho inseguro e com demandas insuportáveis fisicamente e a relação com o estado clinico dos pacientes que se caracterizam como muito graves exigindo maior atenção ao longo do cuidado oferecido. Citam também que as impossibilidades de cura dos pacientes causam temor e riscos de contaminação e a união das equipes deve ser um suporte para estes momentos de angústia vividos por todos.

Além disso, trabalhar na linha de frente com a COVID 19 exige em parte que as equipes sejam capacitadas para atender os pacientes tão descompensados clinicamente que dão entrada nos setores como UTI e emergência. A situação em trabalhar com a pandemia traz elementos adicionais no enfrentamento da realidade e leva ao desequilíbrio físico e mental dos profissionais que atuam diretamente neste contexto, propiciando os sinais e sintomas da síndrome de burnout.

O estudo A10 objetivou analisar a prevalência de sintomas depressão, ansiedade e fatores associados em profissionais da equipe de enfermagem durante a pandemia da Covid-19. Foi realizada com 490 com profissionais de enfermagem dos serviços de média e alta complexidade em um estado do nordeste do Brasil. Neste estudo, houve a apresentação de sintomas sugestivos de transtornos mentais e ansiedade e depressão em profissionais que compuseram a amostra, as condições de trabalho oferecidas especialmente na pandemia foram desencadeadoras dos sinais da síndrome de burnout.

Xiau et al. (2021) reforçam os dados do estudo A9 e A10 quando relatam que as manifestações de quem trabalha na linha de frente no COVID 19 são significativamente resultantes do estresse, pois é evidente o cansaço mental vivido na experiência diária desses profissionais. Muita pressão e o isolamento são fatores que acrescentam a impossibilidade de descanso mental. Os longos turnos impedem de fazer intervalos e os movimentos de retirar EPIs e colocar EPIs, ou ainda permanecer em áreas fechadas e reservadas é gerador de doenças.

Rossi et al. (2019) referem que, para as longas jornadas, deve haver a união da equipe. Além disso, suporte mútuo e 
compreensão mútua, devem estar presentes no cotidiano de trabalho. São fatores que se não estiveram ao alcance das equipes de enfermagem, a falta poderá ser considerada como desencadeador da doença mental. Assim, os achados deste estudo corroboram com A9 A10 quando revelam que as indicações de cuidados com as equipes, como: repouso e intervalos com escalas diferenciadas, adequações de rotinas e espaços físicos, além da oferta de apoio emocional às equipes.

\section{Considerações Finais}

A síndrome de burnout pode ser mais prevalente em seus sinais e sintomas durante a pandemia entre os profissionais de enfermagem. Os estudos levantados discutiram os preditores para a doença e destacaram inúmeros fatores que funcionam como gatilho para que a doença se manifeste.

A síndrome de burnout teve como sinais mais destacadas entre os profissionais a depressão e angústia, por estarem continuamente lidando com as demandas de exigências dos pacientes que são complexos e graves. Além disso, os estudos levantaram que essa síndrome pode ser desencadeada por fatores associados ao ambiente de trabalho, como a falta de recursos humanos, de estrutura física e de valorização por parte dos gestores e empregadores.

Tais resultados encontrados foram acompanhados de sugestões sobre as estratégias que podem ser uteis para minimizar os momentos de desgastes tanto físico quanto mental vividos pelos profissionais de enfermagem, como turnos em rodízios e locais mais apropriados para descanso durante os turnos de trabalho.

Constata-se que os resultados desta revisão integrativa se assemelham com os achados das publicações científicas consultadas. Desse modo, reconhece-se a relevância de pesquisas na área, contribuindo para ampliar os estudos sobre o tema. Recomenda-se novos estudos nessa temática, sobretudo de intervenções para minimizar os danos psicológicos gerados pela pandemia a esses profissionais.

\section{Referências}

Agência Nacional De Vigilância Sanitária. (2020). Covid-19: prevenção e controle durante cirurgias. http://portal.anvisa.gov.br/noticias//asset_publisher/fxrpx9qy7fbu/content/covid-19-prevencao-e-controle-durante-cirurgias/219201/pop_up?

Almeida, I. M. (2020). Proteção da saúde dos trabalhadores da saúde em tempos de pandemia e respostas à pandemia. Revista Brasileira de Saúde Ocupacional. 45(17): 1-10.

Baldonedo-Mosteiro M., Almeida M. C. S., Baptista, B. C. P., Zabalod, M. S., Dias, F. J. R., \& Dias, M. P. M. (2019). Síndrome de burnout em trabalhadores de enfermagem brasileiros e espanhóis. Rev. Latino-Am. Enfermagem, Ribeirão Preto.27 e3192.

Borges, F. E., Borges, A. D., Borges, F. E., Borges, F. E., Sousa, A. S., \& Machado, A. L. (2021). Fatores de risco para a Síndrome de Burnout em profissionais da saúde durante a pandemia de COVID-19. REAID. 95(33):21006.

Conselho federal de enfermagem. (2020).COVID-19. http://www.cofen.gov.br/cofen-disponibiliza-canal-para-ajuda-emocional-a-rofissionais_78283.html

Dal'Bosco, E. B., Floriano, L. S. M., Skupien, S. V., Arcaro, G., Martins, A. R., \& Anselmo, A. C. C. (2020). Mental health of nursing in coping with COVID19 at a regional university hospital. Rev Bras Enferm. 73 (Suppl 2):20200434.

Faro, A., Bahiano, M. A., Nakano, T. C., Reis, C., Silva, B. F. P., \& Santos, L. (2020). COVID-19 e saúde mental: a emergência do cuidado. Estudos de Psicologia (Campinas).37 e200074.

Freitas, R. F., Barros, I. M., Freitas, M. A., Ferreira, M. T., Santos, J. B., \& Less, A. C. (2021). Preditores da síndrome de Burnout em técnicos de enfermagem de unidade de terapia intensiva durante a pandemia da COVID-19. J Bras Psiquiatr. 12-20.

Galea, M. (2014). Avaliando a validade incremental de espiritualidade na previsão do Burnout das enfermeiras. Arch Psychol. Religião. 36(1):118-36.

Gil, A. C. (2010). Métodos e Técnicas de Pesquisa Social, (5a ed.), Atlas.

Horta, R. L., Camargo, E. G., Barbosa, M. L. L., Lantin, P. J. S., Sette, T. G., Lucini, T. G. C., Silveira, A. F., Zanini, L., \& Lutzky, B. A. (2021). O estresse e a saúde mental de profissionais da linha de frente da COVID-19 em hospital geral. J Bras Psiquiatr.70(1):30.

Luz, L. M., Torres, R. R. B., Sarmento, K. M. V. Q., Sales, J. M. R., Farias, K. N., \& Marques, M. B. (2017). Síndrome de burnout em profissionais do serviço de atendimento móvel de urgência. Rev Pesqui Cuid é Fundam. 9(1):238-46.

Medeiros-Costa, M. E., Maciel, R. H., Rêgo, D. P., Lima, L. L., Silva, M. E. P., \& Freitas, J. G. (2017). Occupational Burnout Syndrome in the nursing context: an integrative literature review. Rev da Esc Enferm da USP.11(51):12. 
Research, Society and Development, v. 10, n. 13, e590101321571, 2021

(CC BY 4.0) | ISSN 2525-3409 | DOI: http://dx.doi.org/10.33448/rsd-v10i13.21571

Mercês, M. C., Lopes, R. A., \& Silva, D. S. (2021). Prevalence of Burnout Syndrome in nursing professionals of basic health care. Rev Fund Care, 9(1):208214.

Miranda, A. R. O., \& Afonso, M. L. (2021). Estresse ocupacional de enfermeiros: uma visão crítica em tempos de pandemia. Brazilian Journal of Development. Curitiba.7(4):34979-35000.

Oliveira, F. P., Mazzaia, M. C., \& Marcolan, J. F. (2015). Symptoms of depression and intervening factors among nurses of emergency hospital services. Acta Paul Enferm.28(3):209-15.

Prado, A. D., Peixoto, B. C., Silva, A. M. B., \& Scalia, L. A. M. (2020). A saúde mental dos profissionais de saúde frente à pandemia do COVID-19: uma revisão integrativa. Rev Eletrônica Acervo Saúde. 46: e4128.

Paiva, L. E. B., Lima, T. C. B., Souza, I. C. S., Pitombeira, S. S. R., \& Arruda, S. C. (2016). Síndrome de burnout em operadores de teleatendimento: o caso de duas empresas de contact center em Fortaleza/CE. ReCaPe - Revista de Carreiras e Pessoas São Paulo. 6(2):216-233.

Pereira, M. D., Torres, E. C., Antunes, P. S. F., \& Costa, C. F. T. (2020). Sofrimento emocional dos Enfermeiros no contexto hospitalar frente à pandemia de COVID-19. Research, Society and Development. 9(8); e67985121.

Rodrigues, N. H., \& Silva, L. G. A. (2020). Gestão da pandemia Coronavírus em um hospital: relato de experiência profissional. J.Nurs.health. 10 (esp):e20104004.

Rossi, R., Socci, V., Pacitti, F., Di lorenzo, G., Di Marco, A., \& Sicarusano, A. (2019). Mental Health Outcomes Among Frontline and Second-Line Health Care Workers During the Coronavirus Disease 2019 (COVID-19) Pandemic in Italy. JAMA Netw Open.5: 185.

Santos, K. M. R., Galvão, M. H. R., Gomes, S. M., Souza, T. A., Medeiros, A. A., \& Barbosa, I. R. (2021). Saúde mental de enfermeiros durante a pandemia. Escola Anna Nery. 25(spe).

Schmidt, B., Crepaldi, M. A., Bolze, D. A. S., Silva, L. N., \& Demenech, L. M. (2020). Impactos na Saúde Mental e Intervenções Psicológicas Diante da Pandemia do Novo Coronavírus (COVID-19). Estud. psicol. 37(e200063).

Vidotti, V., Ribeiro, R. P., Galdino, J. Q., \& Martin, J. T. (2018). Síndrome de Burnout e o trabalho em turnos na equipe de enfermagem. Rev. Latino-Am. Enfermagem, Ribeirão Preto.26 e3022.

Xavier, A. R., Ribeiro, R. P., Quina, M. J., Galdino, J.; \& Trevisan, M. (2020). COVID-19: manifestações clínicas e laboratoriais na infecção pelo novo coronavírus. J. Bras. Patol. Med. Lab., Rio de Janeiro.56, e3232020.

Xiao, X., Zhu, X., Fu, S., Hu, Y., Li, X., \& Xiao, J. (2020). Psychological impact of healthcare workers in China during COVID-19 pneumonia epidemic: A multi-center cross-sectional survey investigation. J Affect Disord Rep.10: 274:405. 